patient to refrain from an avoidance behaviour for the triggers, thus improving quality of life and social functioning.

\section{ORGANOPHOSPHORUS PESTICIDE-INDUCED NEUROTOXICITY IN HUMAN APPLICATORS AND ANIMALS WITH COMPARABLE EXPOSURES}

W Kent Anger. Oregon Health and Science University, Portland, OR, USA

10.1136/oemed-2018-ICOHabstracts.818

Aim of special session Preventable chlorpyrifos exposures produce durable behavioural deficits in humans and animals; animal studies suggest oxidative stress contributes to cognitive deficits.

James R. Olson ${ }^{1}$, Diane S. Rohlman ${ }^{2}$, W. Kent Anger ${ }^{3}$, Pamela J. Lein ${ }^{4}$, Fayssal M. Farahat ${ }^{5}$

${ }^{1}$ University at Buffalo, Buffalo, NY, USA

${ }^{2}$ University of Iowa, Iowa City, IA, USA

${ }^{3}$ Oregon Health \& Science University, Portland, OR, USA

${ }^{4}$ University of California Davis, Davis, CA, USA

${ }^{5}$ Menoufia University, Shebin El-Kom, Egypt

\section{9a BIOMARKERS OF CHLORPYRIFOS AND PROFENOFOS EXPOSURE AND EFFECT IN ADULT AND ADOLESCENT EGYPTIAN COTTON FIELD WORKERS}

JR Olson. University at Buffalo, Buffalo, NY, USA

10.1136/oemed-2018-ICOHabstracts.819

Introduction Chlorpyrifos (CPF) and profenofos (PFF) are organophosphorus (OP) pesticides that are applied seasonally in Egypt to cotton fields. Urine trichloro-2-pyridinol (TCPy), a specific CPF metabolite, and 4-bromo-2-chlorophenol (BCP), a specific PFF metabolite, are biomarkers of exposure, while inhibition of blood butyrylcholinesterase (BChE) and acetylcholinesterase (AChE) activities are effect biomarkers which may be associated with neurotoxicity.

Methods Urine TCPy and BCP and blood BChE and AChE activities were measured in 37 adult Egyptian Ministry of Agriculture workers during and after 9-17 consecutive days of CPF application followed by PFF (8-10 days), and a second CPF application (5 days) in 2008. In a separate longitudinal study, 57 adolescent applicators and 38 age-matched nonapplicators were studied over 10 months in 2010 .

Results During the OP applications, mean TCPy and BCP levels were significantly higher than baseline levels and remained elevated following the application periods. Peak urinary BCP and peak TCPy levels for individuals (ranging from 13.4 to 8052 and 16.4 to $30,107 \mathrm{ug} / \mathrm{g}$ creatinine, respectively) were also highly correlated $(\mathrm{r}=0.77, \mathrm{p}<0.001)$. In adults, a significant inverse correlation was observed between urinary TCPy and blood BChE and AChE activities. In the adolescent study, the mean peak TCPy levels were less than the adults, but the exposure-effect relationship for BuChE inhibition was similar to adults. Both adolescent groups had elevated TCPy and depressed BChE which persisted for 4-7 weeks after spraying ended.

Conclusion Biomarker data in the adolescent non-applicators, which mirrored that of the applicators, indicated that the non-applicators received environmental CPF exposures. The variability in environmental and occupational exposures suggest that job title and work location should not be used as the sole basis for categorising OP exposures. Together, these results can serve to guide future investigations in assessing health risks of OPs and guide efforts to reduce exposures.

\section{$1639 \mathrm{~b}$ OCCUPATIONAL PESTICIDE EXPOSURE AMONG EGYPTIAN ADOLESCENTS: CHANGES OVER TIME}

DS Rohlman. University of lowa, lowa City, lowa, USA

\subsection{6/oemed-2018-ICOHabstracts.820}

Introduction While the impact of acute high exposures (i.e., poisoning) to organophosphorus insecticides is well understood, the impact of low level exposures, particularly on neurobehavioral functioning, is still under debate. Even less information is available regarding cumulative exposure, particularly among adolescents who may be working in agriculture. The goal was to examine the impact of chlorpyrifos exposure on biomarkers of exposure and neurobehavioral performance in adolescents across an application season.

Methods Male Egyptian adolescents (applicators and non-applicators) were assessed 35 times before, during and after the pesticide application season. At each session, participants $(n=89)$ completed a neurobehavioral test battery and urine was collected for analysis of the chlorpyrifos metabolite 3,5,6trichloro-2 pyridinol (TCPy) (biomarker of exposure). Cumulative urinary TCPy over the study period was used to classify participants into low $(<$ median $)$ and high $(\geq$ median $)$ exposure groups.

Results Urinary TCPy increased during application with recovery following the end of application. High exposed participants had significantly elevated metabolite levels throughout the 10 month study period. Deficits in motor skills and slower reaction times, along with deficits in executive function and short-term memory were found between the high and low exposure groups. Changes in neurobehavioral performance across the application season indicate a pattern of impaired performance among the high exposed compared to the low exposed. Deficits increased during the application season and remained for months after application ended.

Conclusion The findings indicate that neurobehavioral deficits increase during the application season, as exposure also increases, and remain after the application ends, even when the biomarkers of exposure are reduced. This is particularly important when considering the developmental changes that occur during adolescence. An intervention to reduce pesticide exposure has been implemented in this population.

(Supported by NIEHS and the Fogarty Institute R21 ES017223 and R01 ES022163, Rohlman).

\section{9c ORGANOPHOSPHORUS PESTICIDE NEUROTOXICITY IN EGYPTIAN APPLICATORS}

WK Anger. Oregon Health and Science University, Portland, OR, USA

\subsection{6/oemed-2018-ICOHabstracts.821}

Introduction Chronic occupational exposure to organophosphorus pesticides (OPs) is consistently associated with deficits on neurobehavioral tests when compared to unexposed groups. However, a dose-response relationship has not been established, leading some to doubt an association between 